\title{
Three dimensional view of arbitrary $q$ SYK models
}

\author{
Sumit R. Das, ${ }^{a}$ Animik Ghosh, ${ }^{a}$ Antal Jevicki ${ }^{b}$ and Kenta Suzuki ${ }^{b}$ \\ ${ }^{a}$ Department of Physics and Astronomy, University of Kentucky, \\ Lexington, KY 40506, U.S.A. \\ ${ }^{b}$ Department of Physics, Brown University, \\ 182 Hope Street, Providence, RI 02912, U.S.A. \\ E-mail: das@pa.uky.edu, animik.ghosh@uky.edu, antal_jevicki@brown.edu, \\ kenta_suzuki@brown.edu
}

ABSTRACT: In [15] it was shown that the spectrum and bilocal propagator of SYK model with four fermion interactions can be realized as a three dimensional model in $A d S_{2} \times S^{1} / Z_{2}$ with nontrivial boundary conditions in the additional dimension. In this paper we show that a similar picture holds for generalizations of the SYK model with $q$-fermion interactions. The $3 \mathrm{D}$ realization is now given on a space whose metric is conformal to $A d S_{2} \times S^{1} / Z_{2}$ and is subject to a non-trivial potential in addition to a delta function at the center of the interval. It is shown that a Horava-Witten compactification reproduces the exact SYK spectrum and a non-standard propagator between points which lie at the center of the interval exactly agrees with the bilocal propagator. As $q \rightarrow \infty$, the wave function of one of the modes at the center of the interval vanish as $1 / q$, while the others vanish as $1 / q^{2}$, in a way consistent with the fact that in the SYK model only one of the modes contributes to the bilocal propagator in this limit.

KEYwords: AdS-CFT Correspondence, Gauge-gravity correspondence, 2D Gravity, 1/N Expansion

ARXIV EPrint: 1711.09839 


\section{Contents}

1 Introduction $\quad 1$

$2 \quad q$ fermion SYK model $\quad 3$

3 The three dimensional model $\quad 6$

$\begin{array}{ll}3.1 \text { The spectrum } & 7\end{array}$

3.2 The two point function 8

4 Comparison of the 3d and SYK propagator $\quad 8$

5 The large $q$ limit $\quad 10$

6 Conclusions 12

\section{Introduction}

The Sachdev-Ye-Kitaev (SYK) model [1-5] has in recent intensive studies [6-16] (for a review see [17]), emerged as a useful toy model for holography. An important aspect of this model, which is absent from other such models of holography is the presence of quantum chaos [18-27], which indicates that a finite temperature version of this model describes black holes. Related models have been studied [28-30] with extensions [31-45] and generalizations in the form of tensor type models [46-72]. Interesting random matrix theory interpretations have been realized in [73-80].

The original SYK model has a four fermion interaction with a random coupling which has a gaussian probability distribution with width $J$. Averaging over the couplings gives rise to a theory with eight fermi interactions with coupling $J^{2}$ and an $O(N)$ symmetry, where $N$ is the number of fermions, making this similar to other vector models. Like other vector models, this is most easily solved at large $N$ by making a change of variables to bilocal fields [81, 82]. For such $O(N)$ models it was proposed in [83] that these bi-local fields in fact provide a bulk construction of the dual higher spin theory [84], with the pair of coordinates in the bi-local combining to provide the coordinates of the emergent AdS space-time. In $d \geq 2$, the proposal of [83] was implemented, with additional nonlocal transformations on external legs [85-87] providing a map between the bi-local and conventional Vasiliev higher spin fields in $A d S_{4}$. For $d=1$ case (as in the SYK model) the simplest identification of the center of mass coordinate and the relative coordinate of the two points of the bilocal indeed provides coordinates of a Poincare patch of lorentzian $A d S_{2}$. In $[8,9]$ the collective field theory of the bilocals was developed, providing a transparent way of obtaining both the bilocal propagator and interactions as well as the Schwarzian theory of the low energy mode. 
The precise bulk dual of the SYK model and its $q$ fermion coupling generalization are still not well understood. It has been conjectured in [88-91] that the gravity sector of this model is the Jackiw-Teitelboim model [92, 93] of dilaton-gravity with a negative cosmological constant, studied in [94], while [95] provides strong evidence that it is actually Liouville theory. (See also [96-100]).

The spectrum of the SYK model is highly nontrivial. The matter sector of these theories contains an infinite tower of particles [6-8]. This is clear from the quadratic action for the bilocal fluctuations and the resulting bilocal propagator. The kinetic term in the action contains all powers of the $A d S_{2}$ laplacian which gives rise to rather complicated form of the residues at the poles of the propagator. The couplings of these particles are likewise very complicated, as is clear from the higher point functions computed in $[13,14]$.

In [15] it was shown for the $q=4$ model that the exact spectrum and the bilocal propagator follows from a three dimensional model. In this $3 \mathrm{D}$ realization (where the additional third dimension is used to parametrize the spectrum as in the KK scheme and HS theories), a scalar field with a conventional kinetic energy term is defined on $A d S_{2} \times I$, where $I=S^{1} / Z_{2}$ is a finite interval with a suitable size. The mass of the scalar field is at the Breitenlohner-Freedman bound [101] of $\mathrm{AdS}_{2}$. The scalar field satisfies Dirichlet boundary conditions at the ends and feels an external delta function potential at the middle of the interval. However, as we will see below, the odd parity modes do not play any role in our construction. This means that one can consider half of the interval with Dirichlet condition at one end, and a nontrivial boundary condition determining the derivative of the field at the other end. ${ }^{1}$ The background can be thought of as coming from the nearhorizon geometry of an extremal charged black hole which reduces the gravity sector to Jackiw-Teitelboim model with the metric in the third direction becoming the dilaton of the latter model [89]. The strong coupling limit of the SYK model corresponds to a trivial metric in the third direction, while at finite coupling this acquires a dependence on the $\mathrm{AdS}_{2}$ spatial coordinate. At strong coupling a Horava-Witten compactification then leads to a spectrum of masses in $A d S_{2}$ which is in exact agreement with the SYK spectrum. More significantly, a non-standard propagator with the end points at the location of the delta function exactly reproduces the SYK bilocal propagator, if the two coordinates in the Poincare patch $A d S_{2}$ are identified with the center of mass and the relative coordinate of the two points of the bilocal. The nontrivial factors which appear in the SYK propagator from residues at the poles now appear as the values of the wave function at the center of $I$.

As expected, this strong coupling propagator is divergent due to the divergent contribution of a mode which can be identified with a reparametrization invariance zero mode in the SYK model. At finite coupling the zero mode is lifted and gives rise to an "enhanced contribution" proportional to $J$. In the three dimensional model [15] we adopted the proposal of $[89,90]$, and show that to order $1 / J$, the poles of the propagator shift in a manner consistent with the explicit results in [7] and the enhanced propagator is reproduced as well.

In this paper we show that such a three dimensional picture holds for generalizations of the SYK model with arbitrary $q$. As shown below, the three dimensional metric on which

\footnotetext{
${ }^{1}$ We thank Edward Witten for a clarification on this point.
} 
the scalar lives is now conformal to $A d S_{2} \times I$. The scalar field is subject to a non-trivial potential in addition to a delta function at the center of the interval. This reproduces the spectrum exactly. Furthermore, the three dimensional propagator whose points lie at the center of $I$ reproduce the arbitrary $q$ SYK propagator up to a factor which depends only on $q$. We also discuss the large $q$ limit in this picture. In the SYK model the spectrum becomes evenly spaced in this limit. However only one mode - the zero mode - contributes to the propagator since the residues at the other poles vanish. In the three dimensional picture, the different modes appear as KK modes. However in the large $q$ limit we find that the normalized wave function at the center of the interval is nonzero for only one of these modes, in a way consistent with the SYK result.

In section 2 we describe the bilocal collective formulation of the model for arbitrary $q$. In section 3 we describe our three dimensional model. Section 4 contains the comparison of the propagator of the three dimensional model with the SYK bilocal propagator. In section 5 we comment on the large $q$ limit. Section 6 contains some concluding remarks.

\section{$2 \quad q$ fermion SYK model}

The model is defined by a hamiltonian, with any even $q$,

$$
H=(i)^{\frac{q}{2}} \sum_{1 \leq i_{1}<i_{2}<\cdots<i_{q} \leq N} j_{i_{1} i_{2} \cdots i_{q}} \chi_{i_{1}} \chi_{i_{2}} \cdots \chi_{i_{q}}
$$

where $\chi_{i}$ are Majorana fermions, which satisfy $\left\{\chi_{i}, \chi_{j}\right\}=\delta_{i j}$. The random coupling has a gaussian distribution with

$$
<j_{i_{1} i_{2} \cdots i_{q}}^{2}>=\frac{J^{2}(q-1) !}{N^{q-1}}
$$

One way to perform the averaging is to use the replica trick. At large $N$, one does not expect a spin glass state in this model [5] so that we can restrict to the replica diagonal subspace [8]. The large $N$ this model is efficiently solved by re-writing the theory in terms of replica diagonal bilocal collective fields $[8,9]$.

$$
\Psi\left(t_{1}, t_{2}\right) \equiv \frac{1}{N} \sum_{i=1}^{N} \chi_{i}\left(t_{1}\right) \chi_{i}\left(t_{2}\right),
$$

where we have suppressed the replica index. The corresponding path-integral is

$$
Z=\int \prod_{t_{1}, t_{2}} \mathcal{D} \Psi\left(t_{1}, t_{2}\right) \mu(\Psi) e^{-S_{\mathrm{col}}[\Psi]},
$$

where $S_{\mathrm{col}}$ is the collective action:

$$
S_{\mathrm{col}}[\Psi]=\frac{N}{2} \int d t\left[\partial_{t} \Psi\left(t, t^{\prime}\right)\right]_{t^{\prime}=t}+\frac{N}{2} \operatorname{Tr} \log \Psi-\frac{J^{2} N}{2 q} \int d t_{1} d t_{2} \Psi^{q}\left(t_{1}, t_{2}\right) .
$$

Here the second term comes from a Jacobian factor due to the change of path-integral variable, and the trace is taken over the bi-local time. One also has an appropriate order 
$\mathcal{O}\left(N^{0}\right)$ measure $\mu$. This action being of order $N$ gives a systematic $G=1 / N$ expansion, while the measure $\mu$ found as in [102] begins to contribute at one-loop level (in $1 / N$ ). As is well known, in the IR, i.e. at strong coupling the kinetic term can be ignored. There is now an emergent reparametrization invariance. In this limit the saddle point equation which follow from $S_{\text {col }}[\Psi]$ has the solution

$$
\Psi_{0}\left(t_{1}, t_{2}\right)=\frac{b}{\left|t_{12}\right|^{\frac{2}{q}}} \operatorname{sgn}\left(t_{12}\right) \quad b^{q}=\frac{\tan \left(\frac{\pi}{q}\right)}{J^{2} \pi}\left(\frac{1}{2}-\frac{1}{q}\right)
$$

where we defined $t_{i j} \equiv t_{i}-t_{j}$.

In the following it will be useful to use the center of mass and relative coordinates

$$
t=\frac{1}{2}\left(t_{1}+t_{2}\right), \quad z=\frac{1}{2}\left(t_{1}-t_{2}\right),
$$

The conformal transformations on $t_{1}, t_{2}$ then give rise to transformations on $t, z$ which are identical to the isometries of $A d S_{2}$ with a metric $d s^{2}=\frac{1}{z^{2}}\left[-d t^{2}+d z^{2}\right]^{2} \quad$ Fluctuations around this critical IR solution, $\Psi_{0}(t, z)$ defined by

$$
\Psi\left(t_{1}, t_{2}\right)=\Psi_{0}\left(t_{1}, t_{2}\right)+\sqrt{\frac{2}{N}} \eta(t, z)
$$

can be expanded as

$$
\eta(t, z)=\int \frac{d \omega}{2 \pi} \int \frac{d \nu}{N_{\nu}} \tilde{\Phi}_{\nu, \omega} u_{\nu, \omega}(t, z)
$$

where

$$
u_{\nu, \omega}(t, z)=\operatorname{sgn}(z) e^{i \omega t} Z_{\nu}(|\omega z|)
$$

with $Z_{\nu}$ are a complete set of modes which diagonalizes the quadratic kernel [6],

$$
Z_{\nu}(x)=J_{\nu}(x)+\xi_{\nu} J_{-\nu}(x), \quad \xi_{\nu}=\frac{\tan (\pi \nu / 2)+1}{\tan (\pi \nu / 2)-1}
$$

Their normalization and completeness relations are given by

$$
\begin{aligned}
& \int_{0}^{\infty} \frac{d x}{x} Z_{\nu}^{*}(x) Z_{\nu^{\prime}}(x)=N_{\nu} \delta\left(\nu-\nu^{\prime}\right) \\
& \int \frac{d \nu}{N_{\nu}} Z_{\nu}^{*}(|x|) Z_{\nu}\left(\left|x^{\prime}\right|\right)=x \delta\left(x-x^{\prime}\right)
\end{aligned}
$$

where the normalization factor $N_{\nu}$ is

$$
N_{\nu}= \begin{cases}(2 \nu)^{-1} & \text { for } \nu=3 / 2+2 n \\ 2 \nu^{-1} \sin \pi \nu & \text { for } \nu=i r,\end{cases}
$$

In all of the above expressions the integral over $\nu$ is a shorthand for an integral over the imaginary axis and a sum over the discrete values $\nu=3 / 2+2 n$. The necessity of both the continuous and the discrete spectrum follows from $\mathrm{SL}(2, \mathbb{R})$ representation theory [103].

\footnotetext{
${ }^{2}$ Note that this could very well be $d S_{2}[7]$.
} 
The quadratic part of the fluctuation action then becomes

$$
S_{(2)} \propto J \int d \nu \int d \omega \tilde{\Phi}_{\nu, \omega}^{\star}\left[\tilde{k}_{c}(\nu, q)-1\right] \tilde{\Phi}_{\nu, \omega}
$$

where

$$
\tilde{k}_{c}(\nu, q)=\frac{1}{k_{c}(h, q)} \quad h=\frac{1}{2}+\nu
$$

and $k_{c}(h, q)$ is the eigenvalue of the bilocal kernel derived in [7],

$$
k_{c}(h, q)=-(q-1) \frac{\Gamma\left(\frac{3}{2}-\frac{1}{q}\right) \Gamma\left(1-\frac{1}{q}\right) \Gamma\left(\frac{h}{2}+\frac{1}{q}\right) \Gamma\left(\frac{1}{2}+\frac{1}{q}-\frac{h}{2}\right)}{\Gamma\left(\frac{1}{2}+\frac{1}{q}\right) \Gamma\left(\frac{1}{q}\right) \Gamma\left(\frac{3}{2}-\frac{1}{q}-\frac{h}{2}\right) \Gamma\left(1-\frac{1}{q}+\frac{h}{2}\right)}
$$

The spectrum is then given by solving $k_{c}(h, q)=1$. Note that $p_{m}=3 / 2$ is an exact solution for all $q$.

The bilocal propagator in $(t, z)$ space can be now derived following the same steps as in $[8,15]$ by substituting the expansion $(2.9)$ and using the $(\nu, \omega)$ space propagator which follows from (2.14),

$$
\mathcal{G}\left(t, z ; t^{\prime}, z^{\prime}\right) \sim-\frac{1}{J} \sum_{m} \int_{-\infty}^{\infty} \frac{d \omega}{2 \pi} e^{-i \omega\left(t-t^{\prime}\right)} \int \frac{d \nu}{N_{\nu}} \frac{Z_{\nu}^{*}(|\omega z|) Z_{\nu}\left(\left|\omega z^{\prime}\right|\right)}{\nu^{2}-p_{m}^{2}}\left(2 p_{m}\right) R\left(p_{m}\right)
$$

where $p_{m}$ denote the solutions of the spectral equation $k_{c}\left(p_{m}+\frac{1}{2}, q\right)=1$. The factor $R\left(p_{m}\right)$ is the residue of the propagator at the poles $\nu=p_{m}$,

and

$$
R\left(p_{m}\right)=\frac{1}{\left(\frac{\partial \tilde{k}_{c}(\nu, q)}{\partial \nu}\right)_{\nu=p_{m}}}
$$

where $H_{n}$ denotes the Harmonic number, and

$$
\frac{\partial \tilde{k}_{c}(\nu, q)}{\partial \nu}=N_{h}\left[H_{-1+\frac{h}{2}+\frac{1}{q}}+H_{\frac{1}{2}-\frac{h}{2}-\frac{1}{q}}-H_{\frac{h}{2}-\frac{1}{q}}-H_{-\frac{1}{2}-\frac{h}{2}+\frac{1}{q}}\right]
$$

$$
N_{h}=\frac{\left(\sin \pi h+\sin \frac{2 \pi}{q}\right) \Gamma\left(\frac{2}{q}\right) \Gamma\left(2-h-\frac{2}{q}\right) \Gamma\left(1+h-\frac{2}{q}\right)}{\pi q \Gamma\left(3-\frac{2}{q}\right)}
$$

The symbol $\int d \nu$ is as usual a shorthand notation for an integral over the imaginary axis and a sum over discrete values $\nu=\frac{3}{2}+2 n$. As in the $q=4$ case, when one performs the $\nu$ integral over the imaginary axis there are two sets of poles, the ones at $\nu= \pm p_{m}$ and at $\nu=\frac{3}{2}+2 n$. The contribution from those latter poles exactly cancel the contribution from the discrete values, and one is finally left with an expression

$$
\mathcal{G}\left(t, z ; t^{\prime}, z^{\prime}\right) \sim-\frac{1}{J}\left|z z^{\prime}\right|^{\frac{1}{2}} \sum_{m} \int_{-\infty}^{\infty} \frac{d \omega}{2 \pi} e^{-i \omega\left(t-t^{\prime}\right)} \frac{Z_{-p_{m}}\left(|\omega| z^{>}\right) J_{p_{m}}\left(|\omega| z^{<}\right)}{N_{p_{m}}} R_{p_{m}}
$$

where $z^{<}\left(z^{>}\right)$is the smaller (larger) of $z, z^{\prime}$.

As expected, the expression (2.21) is divergent since this is a strong coupling proagator. This comes from the mode at $p_{m}=3 / 2$ which is a solution for all $q$. At this value $Z_{-3 / 2}$ diverges because $\xi_{-3 / 2}$ diverges. For finite $J$ this mode is corrected by a term which is $O(1 / J)$ and this leads to a contribution to the propagator which is $O(J)$ compared to the contribution from the other solutions of $k_{c}\left(p_{m}+1 / 2, q\right)=1$. 


\section{The three dimensional model}

We will now write down a model which reproduces the above spectrum exactly and the above propagator up to a function of $q$. The model is that of a single scalar field $\Phi$ with an action

$$
\frac{1}{2} \int d t d z d x \sqrt{-g}\left[-g^{\mu \nu} \partial_{\mu} \Phi \partial_{\nu} \Phi-V(x) \Phi^{2}\right]
$$

where the background metric is given by

$$
d s^{2}=|x|^{\frac{4}{q}-1}\left[\frac{-d t^{2}+d z^{2}}{z^{2}}+\frac{d x^{2}}{4|x|(1-|x|)}\right]
$$

and the direction $x$ lies in the interval $-1<x<1$. The space-time is then conformal to $A d S_{2} \times S^{1} / Z_{2}$. The potential which appears in (3.1) is given by

$$
V(x)=\frac{1}{|x|^{\frac{4}{q}-1}}\left[4\left(\frac{1}{q}-\frac{1}{4}\right)^{2}+m_{0}^{2}+\frac{2 V}{J(x)}\left(1-\frac{2}{q}\right) \delta(x)\right]
$$

where $V$ is a constant to be determined below and

$$
J(x)=\frac{|x|^{\frac{2}{q}-1}}{2 \sqrt{1-|x|}}
$$

The action can be now re-written as

$$
\begin{aligned}
S=\frac{1}{2} \int & d t d z d x J(x)\left[\left(\partial_{t} \Phi\right)^{2}-\left(\partial_{z} \Phi\right)^{2}-\frac{m_{0}^{2}}{z^{2}} \Phi^{2}\right. \\
& \left.-\frac{4}{z^{2}}\left\{|x|(1-|x|)\left(\partial_{x} \Phi\right)^{2}+\left(\frac{1}{q}-\frac{1}{4}\right)^{2} \Phi^{2}+\left(1-\frac{2}{q}\right) \frac{V}{2 J(x)} \delta(x) \Phi^{2}\right\}\right]
\end{aligned}
$$

We will impose Dirichlet boundary conditions at $x= \pm 1$,

$$
\Phi(t, z, \pm 1)=0
$$

while the delta function discontinuity in the potential determines the discontinuity at $x=0$ to be

$$
\operatorname{Lim}_{\epsilon \rightarrow 0}\left[|x|^{2 / q} \sqrt{1-|x|} \partial_{x} \Phi\right]_{-\epsilon}^{\epsilon}=\left(1-\frac{2}{q}\right) V \Phi(t, z, 0)
$$

In the following we will be interested in fields which are even under $x \rightarrow-x$. For such fields (3.7) implies

$$
\left[x^{2 / q} \partial_{x} \Phi\right]_{x=0}=\left(1-\frac{2}{q}\right) \frac{V}{2} \Phi(t, z, 0)
$$

Once we impose this we can restrict to $0<x<1$ and forget about the delta function. This is what we will do in the rest of the paper.

Performing an integration by parts and ignoring the boundary term the action becomes

$$
S=\frac{1}{2} \int_{-\infty}^{\infty} d t \int_{0}^{\infty} d z \int_{0}^{1} d x J(x) \Phi \mathcal{D}_{0} \Phi
$$

where

$$
\mathcal{D}_{0}=-\partial_{t}^{2}+\partial_{z}^{2}-\frac{m_{0}^{2}}{z^{2}}+\frac{4}{z^{2}}\left[x(1-x) \partial_{x}^{2}+\left[\frac{2}{q}-x\left(\frac{1}{2}+\frac{2}{q}\right)\right] \partial_{x}-\left(\frac{1}{q}-\frac{1}{4}\right)^{2}\right]
$$

This operator is hermitian with the measure $d x J(x)$. 


\subsection{The spectrum}

To diagonalize $\mathcal{D}_{0}$ we first find solve the eigenvalue problem for the operator inside the square bracket in (3.10) in the domain $0<x<1$,

$$
\left[x(1-x) \partial_{x}^{2}+\left[\frac{2}{q}-x\left(\frac{1}{2}+\frac{2}{q}\right)\right] \partial_{x}-\left(\frac{1}{q}-\frac{1}{4}\right)^{2}\right] \phi_{k}(x)=-\frac{k^{2}}{4} \phi_{k}(x)
$$

The general solution of this equation is

$$
\phi_{k}(x)=A_{2} F_{1}(a, b ; c, x)+x^{1-c} B{ }_{2} F_{1}(a-c+1, b-c+1 ; 2-c ; x)
$$

where ${ }_{2} F_{1}$ denotes the usual Hypergeometric function and

$$
a=\frac{1}{q}-\frac{1}{4}-\frac{k}{2} \quad b=\frac{1}{q}-\frac{1}{4}+\frac{k}{2} \quad c=\frac{2}{q}
$$

Imposing the boundary condition (3.8) gives

$$
B=\frac{V}{2} A
$$

while imposing (3.6) gives

$$
A_{2} F_{1}(a, b ; c, 1)+B_{2} F_{1}(a-c+1, b-c+1 ; 2-c ; 1)=0
$$

Using

$$
{ }_{2} F_{1}(a, b ; c, 1)=\frac{\Gamma(c) \Gamma(c-a-b)}{\Gamma(c-a) \Gamma(c-b)}
$$

and combining (3.15) and (3.14) we get

$$
\frac{\Gamma\left(\frac{5}{4}-\frac{1}{q}-\frac{k}{2}\right) \Gamma\left(\frac{2}{q}\right) \Gamma\left(\frac{5}{4}-\frac{1}{q}+\frac{k}{2}\right)}{\Gamma\left(2-\frac{2}{q}\right) \Gamma\left(\frac{1}{4}+\frac{1}{q}-\frac{k}{2}\right) \Gamma\left(\frac{1}{4}+\frac{1}{q}+\frac{k}{2}\right)}=-\frac{V}{2}
$$

where we have used the values of $a, b, c$ in (3.13).

Remarkably if we choose

$$
V=2(q-1) \frac{\Gamma\left(\frac{3}{2}-\frac{1}{q}\right) \Gamma\left(1-\frac{1}{q}\right) \Gamma\left(\frac{2}{q}\right)}{\Gamma\left(\frac{1}{2}+\frac{1}{q}\right) \Gamma\left(2-\frac{2}{q}\right) \Gamma\left(\frac{1}{q}\right)}
$$

and define $h=k+1 / 2$ the condition (3.17) becomes

$$
k_{c}(h, q)=1
$$

where $k_{c}(h, q)$ is precisely the SYK spectrum for arbitrary $q$ given by (2.16). The significant point of course is that $V$ given by (3.18) depends only on $q$. 


\subsection{The two point function}

Using the eigenfunctions in the previous subsection we can now expand the three dimensional field in terms of a complete basis as follows

$$
\Phi(t, z, x)=\int \frac{d k d \nu d \omega}{N_{\nu}} e^{-i \omega t}|z|^{1 / 2} Z_{\nu}(|\omega z|) \varphi_{k}(x) \chi(\omega, \nu, k)
$$

where the combinations of Bessel functions $Z_{\nu}$ have been defined in $(2.11)$ and $\varphi_{k}(x)$ are

$$
\varphi_{k}(x)={ }_{2} F_{1}(a, b ; c, x)+x^{1-c} \frac{V}{2}{ }_{2} F_{1}(a-c+1, b-c+1 ; 2-c ; x)
$$

where $a, b, c$ are given in (3.13) and $V$ is given in (3.18). The functions $\varphi_{k}(x)$ are orthogonal with the measure factor $J(x)$

$$
\int_{0}^{1} d x J(x) \varphi_{k}(x) \varphi_{k^{\prime}}(x)=C_{1}(k) \delta_{k, k^{\prime}}
$$

The action now becomes

$$
S=\frac{1}{2} \int \frac{d k d \nu d \omega}{N_{\nu}} C_{1}(k)\left(\nu^{2}-\nu_{0}^{2}\right) \chi(\omega, \nu, k) \chi(-\omega, \nu, k)
$$

where

$$
\nu_{0}^{2}=k^{2}+m_{0}^{2}+\frac{1}{4}
$$

Let us now choose

$$
m_{0}^{2}=-1 / 4
$$

so that one finally has $\nu_{0}=k$. The two point function of $\chi(\omega \nu k)$ is then given by

$$
\langle\chi(\omega, \nu, k) \chi(-\omega, \nu, k)\rangle=\frac{N_{\nu}}{C_{1}(k)\left(\nu^{2}-k^{2}\right)}
$$

The position space $3 \mathrm{~d}$ propagator is then given by

$$
\left\langle\Phi(t, z, x) \Phi\left(t^{\prime}, z^{\prime}, x^{\prime}\right)\right\rangle=\left|z z^{\prime}\right|^{\frac{1}{2}} \sum_{m} C\left(p_{m}, x, x^{\prime}\right) \int \frac{d \omega}{2 \pi} e^{-i \omega\left(t-t^{\prime}\right)} \int \frac{d \nu}{N_{\nu}} \frac{Z_{\nu}^{\star}(|\omega z|) Z_{\nu}\left(\left|\omega z^{\prime}\right|\right)}{\nu^{2}-p_{m}^{2}}
$$

where

$$
C\left(p_{m}, x, x^{\prime}\right)=\frac{\varphi_{p_{m}}(x) \varphi_{p_{m}}\left(x^{\prime}\right)}{C_{1}\left(p_{m}\right)}
$$

This can be regarded as a sum of $A d S_{2}$ propagators. However it is important to note that these are non-standard propagators.

\section{Comparison of the $3 \mathrm{~d}$ and SYK propagator}

We now show that the propagator (3.27) evaluated at $x=x^{\prime}=0$ agrees with the SYK propagator (2.17) multiplied by a factor of $\left(z z^{\prime}\right)^{1 / 2}$, up to an overall factor which depends on $q$. The values of $p_{m}$ over which the two expressions need to be summed have been 


\begin{tabular}{|c|c|c|c|c|}
\hline$q$ & $p_{m}$ & $C\left(p_{m}, 0,0\right)$ & $2 p_{m} R\left(p_{m}\right)$ & $\frac{C\left(p_{m}, 0,0\right)}{2 p_{m} R\left(p_{m}\right)}$ \\
\hline 6 & 1.5 & 0.415724 & 1.09987 & 0.377976 \\
& 3.07763 & 0.566693 & 1.49928 & 0.377976 \\
& 4.95427 & 0.474406 & 1.25512 & 0.377976 \\
& 6.90849 & 0.409177 & 1.08255 & 0.377976 \\
& 8.88613 & 0.366967 & 0.970874 & 0.377976 \\
\hline 8 & 1.5 & 0.344227 & 1.27788 & 0.269374 \\
& 2.95416 & 0.357211 & 1.32608 & 0.269374 \\
& 4.835 & 0.241026 & 0.894764 & 0.269374 \\
& 6.79849 & 0.188249 & 0.698838 & 0.269374 \\
& 8.78225 & 0.159144 & 0.590793 & 0.269374 \\
\hline 12 & 1.5 & 0.256089 & 1.47882 & 0.173171 \\
& 2.81505 & 0.175464 & 1.01324 & 0.173171 \\
& 4.71763 & 0.0925242 & 0.534294 & 0.173171 \\
& 6.69343 & 0.0660067 & 0.381165 & 0.173171 \\
& 8.68356 & 0.0529405 & 0.305712 & 0.173171 \\
\hline 20 & 1.5 & 0.169337 & 1.66312 & 0.101819 \\
& 2.69405 & 0.0678495 & 0.666375 & 0.101819 \\
& 4.62734 & 0.0287883 & 0.28274 & 0.101819 \\
& 6.61348 & 0.0192516 & 0.189077 & 0.101819 \\
& 8.60817 & 0.0148617 & 0.145963 & 0.101819 \\
\hline 50 & 1.5 & 0.0745898 & 1.85438 & 0.0402235 \\
& 2.57914 & 0.0115317 & 0.286691 & 0.0402235 \\
& 4.54971 & 0.00396415 & 0.0985529 & 0.0402235 \\
& 6.54453 & 0.00251532 & 0.0625335 & 0.0402235 \\
\hline
\end{tabular}

Table 1. Comparison of factors appearing in the 3d and SYK propagators.

already seen to be identical, so we need to compare the coefficients which appear. To compare (2.17) and (3.27) we need to compute the quantity

$$
\frac{C\left(p_{m}, 0,0\right)}{2 p_{m} R\left(p_{m}\right)}
$$

and show that this is independent of $p_{m}$. Here

$$
C_{1}\left(p_{m}\right)=\int_{0}^{1} d x J(x) \varphi_{p_{m}}(x) \varphi_{p_{m}}(x)
$$

We have not been able to evaluate this integral analytically, but have performed this numerically to high precision. In table 1 we tabulate the values of the relevant quantities for various values of $q$ and $p_{m}$ which solve the spectrum equation, and compare them with the corresponding factors which appear in (2.17)

For a given value of $q$ the value of the ratio (4.1) is independent of $p_{m}$ upto 13 decimal places. This shows that this ratio is only a function of $q$ which we denote by $f(q)$. These results show that for any given $q$, the non-standard propagator of the $3 \mathrm{~d}$ model with the 
two points at $x=0$ is proportional to the SYK propagator. The data also shows that $f(q)$ decreases with $q$.

As for $q=4$, the propagator (3.27) is actually divergent from the contribution of the $p_{m}=3 / 2$ mode, as expected from the SYK propagator at infinite coupling. We expect that a modification of the three dimensional background would reproduce the enhanced propagator of this mode, as happened for $q=4[15]$.

\section{$5 \quad$ The large $q$ limit}

In the $q \rightarrow \infty$ limit, $p_{m}=3 / 2$ remains a solution, while the other solutions of the spectral equation (2.16) become very simple,

$$
p_{m}=2 m+\frac{1}{2}+\frac{2}{q} \frac{2 m^{2}+m+1}{2 m^{2}+m-1}+\cdots \quad m=1,2, \cdots
$$

To calculate the contribution to these poles to the SYK propagator consider the residue $R\left(p_{m}\right)$ in (2.18). In a $1 / q$ expansion we find that for $p_{m}=3 / 2$

$$
R(3 / 2)=\frac{2}{3}-\frac{1}{q}\left(\frac{5}{2}+\frac{\pi^{2}}{3}\right)+O\left(1 / q^{2}\right)
$$

while for the other solutions we get ${ }^{3}$

$$
R\left(p_{m}\right) \rightarrow \frac{1}{q} \frac{4\left(2 m^{2}+m\right)}{\left(2 m^{2}+m-1\right)^{2}}+O\left(1 / q^{2}\right)
$$

Thus only the pole at $p_{m}=3 / 2$ has a non-vanishing residue in the large $q$ limit. Of course the strong coupling propagator is infinite from contribution of the $p_{m}=3 / 2$ mode. However a finite $J$ correction would lead to a nonzero contribution proportional to $J$ [7].

In the three dimensional picture this happens because of the different large $q$ behavior of the wave function at $x=0$ for $p_{m}=3 / 2$ compared to the other values of $p_{m}$. For large enough $q$ we can use $p_{m}=2 m+1$ as a good approximation to the solution of the spectral equation. In table 2 we tabulate the values of the square of the wave function at $x=x^{\prime}=0$, i.e. $C\left(p_{m}, 0,0\right)$, the value of the quantity $2 p_{m} R\left(p_{m}\right)$ which appears in the SYK propagator and the quantity $q f(q)$ where

$$
f(q)=\frac{C\left(p_{m}, 0,0\right)}{2 p_{m} R\left(p_{m}\right)}
$$

for large values of $q$, for different values of $m$. We have checked that for the values of $q$ which we have used, $p_{m}=2 m+1$ is indeed a very good approximation to the exact solution of $k_{c}(h, q)=1$. We then tabulate this quantity for given $m$ for various values of $q$. The results show that $q f(q)$ is a constant to very high accuracy. Using (5.2) and (5.3) we then conclude that the wave function at $x=0$ for $p_{m} \neq 3 / 2$ modes vanishes as $\frac{1}{q^{2}}$, while that for the $p_{m}=3 / 2$ this vanishes as $\frac{1}{q}$.

A plot of $f(q)$ for $p_{m}=3 / 2$ is given in figure 1 .

\footnotetext{
${ }^{3}$ We thank Pranjal Nayak for this calculation.
} 


\begin{tabular}{|c|c|c|c|c|}
\hline$p_{m}=2 m+1 / 2$ & $q$ & $C_{1}\left(p_{m}, 0,0\right)$ & $2 p_{m} R\left(p_{m}\right)$ & $\frac{q C\left(p_{m}, 0,0\right)}{2 p_{m} R\left(p_{m}\right)}$ \\
\hline $3 / 2$ & 500 & 125.908 & 1.98465 & 2.00093 \\
& 600 & 150.908 & 1.9872 & 2.00077 \\
& 800 & 200.908 & 1.99039 & 2.00058 \\
& 1000 & 250.908 & 1.9923 & 2.00046 \\
\hline $5 / 2$ & 500 & 74442.2 & 0.003356 & 2.00093 \\
& 600 & 107330 & 0.002794 & 2.00077 \\
& 800 & 191107 & 0.002092 & 2.00058 \\
& 1000 & 298883 & 0.00167 & 2.00046 \\
\hline $9 / 2$ & 500 & 137225 & 0.00182 & 2.00093 \\
& 600 & 198038 & 0.00151 & 2.00077 \\
& 800 & 352937 & 0.001133 & 2.00058 \\
& 1000 & 552280 & 0.000905 & 2.00046 \\
\hline $21 / 2$ & 500 & 321462 & 0.00077 & 2.00093 \\
& 600 & 464316 & 0.000645 & 2.00077 \\
& 800 & 828595 & 0.000484 & 2.00058 \\
& 1000 & $1.279 \times 10^{6}$ & 0.000385 & 2.00084 \\
\hline $41 / 2$ & 500 & 625414 & 0.000399 & 2.00093 \\
& 600 & 904123 & 0.000331 & 2.00077 \\
& 800 & $1.615 \times 10^{6}$ & 0.000247 & 2.00082 \\
& 1000 & $2.52 \times 10^{6}$ & 0.000197 & 2.00065 \\
\hline & & & & \\
& & & \\
& & & \\
& 500 &
\end{tabular}

Table 2. Large-q behavior of the wave function at $x=x^{\prime}=0$ for different values of $p_{m}$.

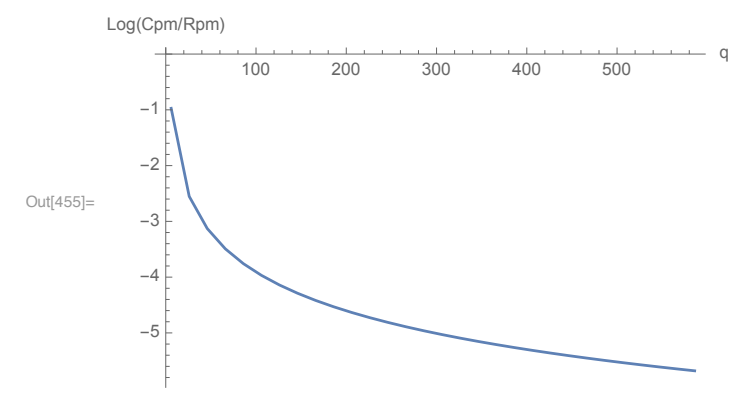

Figure 1. Plot of $\log f(q)$ for $p_{m}=3 / 2$. For large $q$ the data fits well with the function $f(q) \sim \frac{2}{q}$.

This behavior provides an understanding of the decoupling of the other modes in the tower at large $q$. Note that the other modes are still present, though they do not contribute to the propagator. In fact the large $q$ limit is subtle. If we perform a $1 / q$ expansion of the collective action for the bilocal field, (2.5), using the parametrization used in [7] one ends up with a Liouville theory in the $(t, z)$ space for all values of the suitably rescaled coupling [104]. ${ }^{4}$ This has a conventional kinetic term - so that one seems to get a single two

\footnotetext{
${ }^{4}$ The Dyson-Schwinger equation in the $q \rightarrow \infty$ limit is already known to be akin to the Liouville equation [7]. Here we are making a stronger statement about the bilocal field itself, not just about its saddle point value.
} 
dimensional field, the corresponding pole of the propagator being precisely the $p_{m}=3 / 2$ mode. The other modes are simply absent in this treatment, and seem to be recovered due to nonlocal $1 / \mathrm{q}$ interactions.

\section{Conclusions}

It is remarkable that the complicated tower of states which appear in the SYK model can be understood as a KK tower in a fixed background for arbitrary $q$. We want to emphasize that while reproducing the spectrum is already quite interesting, the agreement of the propagator with the bilocal propagator is highly non-trivial. This gives a strong evidence that a three dimensional space-time is an essential ingredient of the full dual to the SYK model.

Unlike the $q=4$ case we do not yet have a natural understanding of the three dimensional background at arbitrary $q$ in terms of a near horizon geometry of a black hole. In fact we arrived at this background by "reverse engineering" (the clue was that ratios of gamma functions come in trying to match hypergeometric functions). We hope to be able to find a natural origin of this background. That will provide a natural way to understand the finite $J$ correction and in particular the enhanced propagator of the $p_{m}=3 / 2$ mode. Furthermore the delta function potential can be possibly interpreted as a brane - it will be interesting to see if such a brane model an be indeed constructed.

In this paper we have not addressed the question of interactions of the bilocals. These have been considered in $[13,14]$ and are expected to follow from the cubic and higher order terms in the collective field theory of [8]. It will be interesting to see what kind of interactions in the three dimensional model reproduce these and investigate their locality (or lack thereof) properties. ${ }^{5}$ An important aspect of the $3 \mathrm{~d}$ picture is that while the propagator can be written as a sum of $A d S_{2}$ propagators, the latter are non-standard propagators. While they do vanish at the boundary, they have different boundary conditions at the Poincare horizon. A second unusual aspect is that the space of bilocals always gives rise to Lorentzian $A d S_{2}$ even if we start out with an euclidean theory. The issues raised above require a better understanding of the bulk theory, we will address them in a forthcoming publication [105].

\section{Acknowledgments}

We thank Juan Maldacena and Pranjal Nayak for discussions, and Ed Witten for a correspondence. This work of AJ and KS is supported by the Department of Energy under contract DE-SC0010010. The work of KS is also supported by the Galkin Fellowship Award at Brown University. The work of SRD and AG is partially supported by the National Science Foundation grant NSF-PHY-1521045. S.R.D. would like to thank Yukawa Institute for Theoretical Physics, Kyoto University and Tata Institute of Fundamental Research, Mumbai for hospitality during the completion of this work. AG would like to thank the lecturers and participants of the TASI 2017 program, and the University of Colorado, Boulder for hospitality during the completion of this work.

\footnotetext{
${ }^{5}$ In [14] a different 3d background is shown to reproduce the large- $q$ spectrum.
} 
Open Access. This article is distributed under the terms of the Creative Commons Attribution License (CC-BY 4.0), which permits any use, distribution and reproduction in any medium, provided the original author(s) and source are credited.

\section{References}

[1] S. Sachdev and J. Ye, Gapless spin fluid ground state in a random, quantum Heisenberg magnet, Phys. Rev. Lett. 70 (1993) 3339 [cond-mat/9212030] [INSPIRE].

[2] S. Sachdev, Holographic metals and the fractionalized Fermi liquid, Phys. Rev. Lett. 105 (2010) 151602 [arXiv:1006.3794] [INSPIRE].

[3] A. Kitaev, Hidden correlations in the hawking radiation and thermal noise, talk given at the Fundamental Physics Prize Symposium, November 10, Stanford University, Stanford, U.S.A. (2014).

[4] A. Kitaev, A simple model of quantum holography, talks given at the KITP strings seminar and Entanglement 2015 program, February 12, April 7, and May 27, KITP, Santa Barbara, U.S.A. (2015).

[5] S. Sachdev, Bekenstein-Hawking entropy and strange metals, Phys. Rev. X 5 (2015) 041025 [arXiv: 1506.05111] [INSPIRE].

[6] J. Polchinski and V. Rosenhaus, The spectrum in the Sachdev-Ye-Kitaev model, JHEP 04 (2016) 001 [arXiv: 1601.06768] [InSPIRE].

[7] J. Maldacena and D. Stanford, Remarks on the Sachdev-Ye-Kitaev model, Phys. Rev. D 94 (2016) 106002 [arXiv: 1604.07818] [INSPIRE].

[8] A. Jevicki, K. Suzuki and J. Yoon, Bi-local holography in the SYK model, JHEP 07 (2016) 007 [arXiv: 1603.06246] [INSPIRE].

[9] A. Jevicki and K. Suzuki, Bi-local holography in the SYK model: perturbations, JHEP 11 (2016) 046 [arXiv: 1608.07567] [INSPIRE].

[10] R.A. Davison, W. Fu, A. Georges, Y. Gu, K. Jensen and S. Sachdev, Thermoelectric transport in disordered metals without quasiparticles: the Sachdev-Ye-Kitaev models and holography, Phys. Rev. B 95 (2017) 155131 [arXiv:1612.00849] [INSPIRE].

[11] D. Stanford and E. Witten, Fermionic localization of the schwarzian theory, JHEP 10 (2017) 008 [arXiv: 1703.04612] [inSPIRE].

[12] T.G. Mertens, G.J. Turiaci and H.L. Verlinde, Solving the Schwarzian via the conformal bootstrap, JHEP 08 (2017) 136 [arXiv:1705.08408] [INSPIRE].

[13] D.J. Gross and V. Rosenhaus, The bulk dual of SYK: cubic couplings, JHEP 05 (2017) 092 [arXiv: 1702.08016] [INSPIRE].

[14] D.J. Gross and V. Rosenhaus, All point correlation functions in SYK, JHEP 12 (2017) 148 [arXiv: 1710.08113] [INSPIRE].

[15] S.R. Das, A. Jevicki and K. Suzuki, Three dimensional view of the SYK/AdS duality, JHEP 09 (2017) 017 [arXiv: 1704.07208] [INSPIRE].

[16] A. Kitaev and S.J. Suh, The soft mode in the Sachdev-Ye-Kitaev model and its gravity dual, arXiv: 1711.08467 [INSPIRE].

[17] G. Sárosi, $A d S_{2}$ holography and the $S Y K$ model, arXiv:1711.08482 [INSPIRE]. 
[18] S.H. Shenker and D. Stanford, Black holes and the butterfly effect, JHEP 03 (2014) 067 [arXiv: 1306.0622] [INSPIRE].

[19] S. Leichenauer, Disrupting entanglement of black holes, Phys. Rev. D 90 (2014) 046009 [arXiv: 1405.7365] [INSPIRE].

[20] S.H. Shenker and D. Stanford, Stringy effects in scrambling, JHEP 05 (2015) 132 [arXiv: 1412.6087] [INSPIRE].

[21] J. Maldacena, S.H. Shenker and D. Stanford, A bound on chaos, JHEP 08 (2016) 106 [arXiv: 1503.01409] [INSPIRE].

[22] J. Polchinski, Chaos in the black hole S-matrix, arXiv:1505.08108 [INSPIRE].

[23] P. Caputa, T. Numasawa and A. Veliz-Osorio, Out-of-time-ordered correlators and purity in rational conformal field theories, PTEP 2016 (2016) 113B06 [arXiv: 1602.06542] [INSPIRE].

[24] Y. Gu and X.-L. Qi, Fractional Statistics and the Butterfly Effect, JHEP 08 (2016) 129 [arXiv:1602.06543] [INSPIRE].

[25] E. Perlmutter, Bounding the space of holographic CFTs with chaos, JHEP 10 (2016) 069 [arXiv: 1602.08272] [INSPIRE].

[26] D. Anninos, T. Anous and F. Denef, Disordered quivers and cold horizons, JHEP 12 (2016) 071 [arXiv: 1603.00453] [INSPIRE].

[27] G. Turiaci and H. Verlinde, On CFT and quantum chaos, JHEP 12 (2016) 110 [arXiv: 1603.03020] [INSPIRE].

[28] I. Danshita, M. Hanada and M. Tezuka, Creating and probing the Sachdev-Ye-Kitaev model with ultracold gases: Towards experimental studies of quantum gravity, PTEP 2017 (2017) 083I01 [arXiv: 1606.02454] [INSPIRE].

[29] J. Erdmenger et al., Holographic impurities and Kondo effect, Fortsch. Phys. 64 (2016) 322 [arXiv: 1511.09362] [INSPIRE].

[30] C. Krishnan, K.V. Pavan Kumar and D. Rosa, Contrasting SYK-like models, JHEP 01 (2018) 064 [arXiv: 1709. 06498] [inSPIRE].

[31] D.J. Gross and V. Rosenhaus, A generalization of Sachdev-Ye-Kitaev, JHEP 02 (2017) 093 [arXiv: 1610.01569] [INSPIRE].

[32] Y. Gu, X.-L. Qi and D. Stanford, Local criticality, diffusion and chaos in generalized Sachdev-Ye-Kitaev models, JHEP 05 (2017) 125 [arXiv:1609.07832] [INSPIRE].

[33] M. Berkooz, P. Narayan, M. Rozali and J. Simón, Higher dimensional generalizations of the SYK model, JHEP 01 (2017) 138 [arXiv:1610.02422] [INSPIRE].

[34] W. Fu, D. Gaiotto, J. Maldacena and S. Sachdev, Supersymmetric Sachdev-Ye-Kitaev models, Phys. Rev. D 95 (2017) 026009 [arXiv:1610.08917] [InSPIRE].

[35] W. Fu and S. Sachdev, Numerical study of fermion and boson models with infinite-range random interactions, Phys. Rev. B 94 (2016) 035135 [arXiv: 1603.05246] [INSPIRE].

[36] L. García-Álvarez et al., Digital quantum simulation of minimal AdS/CFT, Phys. Rev. Lett. 119 (2017) 040501 [arXiv: 1607.08560] [INSPIRE].

[37] S.A. Hartnoll, L. Huijse and E.A. Mazenc, Matrix quantum mechanics from qubits, JHEP 01 (2017) 010 [arXiv: 1608.05090] [INSPIRE]. 
[38] T. Nishinaka and S. Terashima, A note on Sachdev-Ye-Kitaev like model without random coupling, Nucl. Phys. B 926 (2018) 321 [arXiv:1611.10290] [INSPIRE].

[39] G. Turiaci and H. Verlinde, Towards a 2d QFT analog of the SYK model, JHEP 10 (2017) 167 [arXiv: 1701.00528] [INSPIRE].

[40] S.-K. Jian and H. Yao, Solvable Sachdev-Ye-Kitaev models in higher dimensions: from diffusion to many-body localization, Phys. Rev. Lett. 119 (2017) 206602 [arXiv: 1703.02051] [INSPIRE].

[41] A. Chew, A. Essin and J. Alicea, Approximating the Sachdev-Ye-Kitaev model with Majorana wires, Phys. Rev. B 96 (2017) 121119 [arXiv:1703.06890] [InSPIRE].

[42] M. Blake and A. Donos, Diffusion and chaos from near AdS 2 horizons, JHEP 02 (2017) 013 [arXiv: 1611.09380] [INSPIRE].

[43] J. Murugan, D. Stanford and E. Witten, More on supersymmetric and $2 d$ analogs of the SYK model, JHEP 08 (2017) 146 [arXiv: 1706. 05362] [INSPIRE].

[44] J. Yoon, Supersymmetric SYK Model: Bi-local Collective Superfield/Supermatrix Formulation, JHEP 10 (2017) 172 [arXiv:1706.05914] [INSPIRE].

[45] W. Cai, X.-H. Ge and G.-H. Yang, Diffusion in higher dimensional SYK model with complex fermions, JHEP 01 (2018) 076 [arXiv:1711.07903] [INSPIRE].

[46] E. Witten, An SYK-like model without disorder, arXiv:1610.09758 [INSPIRE].

[47] R. Gurau, The complete $1 / N$ expansion of a SYK-like tensor model, Nucl. Phys. B 916 (2017) 386 [arXiv: 1611.04032] [INSPIRE].

[48] R. Gurau, The $1 / N$ expansion of tensor models with two symmetric tensors, Commun. Math. Phys. (2017) [arXiv:1706.05328] [INSPIRE].

[49] I.R. Klebanov and G. Tarnopolsky, Uncolored random tensors, melon diagrams and the Sachdev-Ye-Kitaev models, Phys. Rev. D 95 (2017) 046004 [arXiv:1611.08915] [InSPIRE].

[50] I.R. Klebanov and G. Tarnopolsky, On large- $N$ limit of symmetric traceless tensor models, JHEP 10 (2017) 037 [arXiv:1706.00839] [INSPIRE].

[51] S. Giombi, I.R. Klebanov and G. Tarnopolsky, Bosonic tensor models at large- $N$ and small

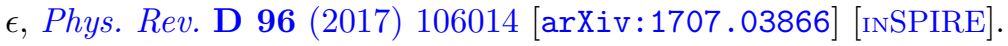

[52] K. Bulycheva, I.R. Klebanov, A. Milekhin and G. Tarnopolsky, Spectra of operators in large-N tensor models, Phys. Rev. D 97 (2018) 026016 [arXiv:1707.09347] [INSPIRE].

[53] C. Peng, M. Spradlin and A. Volovich, A supersymmetric SYK-like tensor model, JHEP 05 (2017) 062 [arXiv: 1612.03851] [InSPIRE].

[54] C. Peng, M. Spradlin and A. Volovich, Correlators in the $\mathcal{N}=2$ supersymmetric SYK model, JHEP 10 (2017) 202 [arXiv:1706. 06078] [INSPIRE].

[55] C. Peng, Vector models and generalized SYK models, JHEP 05 (2017) 129 [arXiv: 1704.04223] [INSPIRE].

[56] F. Ferrari, The large D limit of planar diagrams, arXiv:1701.01171 [INSPIRE].

[57] T. Azeyanagi, F. Ferrari and F.I. Schaposnik Massolo, Phase diagram of planar matrix quantum mechanics, tensor and Sachdev-Ye-Kitaev models, Phys. Rev. Lett. 120 (2018) 061602 [arXiv: 1707.03431] [INSPIRE]. 
[58] H. Itoyama, A. Mironov and A. Morozov, Rainbow tensor model with enhanced symmetry and extreme melonic dominance, Phys. Lett. B 771 (2017) 180 [arXiv:1703.04983] [INSPIRE].

[59] H. Itoyama, A. Mironov and A. Morozov, Ward identities and combinatorics of rainbow tensor models, JHEP 06 (2017) 115 [arXiv: 1704.08648] [INSPIRE].

[60] A. Mironov and A. Morozov, Correlators in tensor models from character calculus, Phys. Lett. B 774 (2017) 210 [arXiv:1706.03667] [INSPIRE].

[61] H. Itoyama, A. Mironov and A. Morozov, Cut and join operator ring in Aristotelian tensor model, arXiv:1710.10027 [INSPIRE].

[62] P. Narayan and J. Yoon, SYK-like tensor models on the lattice, JHEP 08 (2017) 083 [arXiv: 1705.01554] [INSPIRE].

[63] S. Chaudhuri et al., Abelian tensor models on the lattice, arXiv:1705.01930 [INSPIRE].

[64] J. Yoon, SYK models AND syk-like tensor models with global symmetry, JHEP 10 (2017) 183 [arXiv: 1707.01740] [INSPIRE].

[65] P. Diaz and S.-J. Rey, Orthogonal bases of invariants in tensor models, JHEP 02 (2018) 089 [arXiv: 1706. 02667] [INSPIRE].

[66] R. de Mello Koch, R. Mello Koch, D. Gossman and L. Tribelhorn, Gauge invariants, correlators and holography in bosonic and fermionic tensor models, JHEP 09 (2017) 011 [arXiv: 1707.01455] [INSPIRE].

[67] S. Choudhury, A. Dey, I. Halder, L. Janagal, S. Minwalla and R. Poojary, Notes on melonic $O(n)^{q-1}$ tensor models, arXiv: 1707.09352 [INSPIRE].

[68] J. Ben Geloun and S. Ramgoolam, Counting tensor model observables and branched covers of the 2-sphere, arXiv:1307.6490 [INSPIRE].

[69] J. Ben Geloun and S. Ramgoolam, Tensor models, Kronecker coefficients and permutation centralizer algebras, JHEP 11 (2017) 092 [arXiv: 1708.03524] [INSPIRE].

[70] S. Prakash and R. Sinha, A complex fermionic tensor model in d dimensions, arXiv: 1710.09357 [INSPIRE].

[71] N. Halmagyi and S. Mondal, Tensor models for black hole probes, arXiv:1711.04385 [INSPIRE].

[72] J. Ben Geloun and V. Rivasseau, A renormalizable SYK-type tensor field theory, arXiv: 1711.05967 [INSPIRE].

[73] Y.-Z. You, A.W.W. Ludwig and C. Xu, Sachdev-Ye-Kitaev model and thermalization on the boundary of many-body localized fermionic symmetry protected topological states, Phys. Rev. B 95 (2017) 115150 [arXiv: 1602.06964] [InSPIRE].

[74] A.M. García-García and J.J.M. Verbaarschot, Spectral and thermodynamic properties of the Sachdev-Ye-Kitaev model, Phys. Rev. D 94 (2016) 126010 [arXiv:1610.03816] [INSPIRE].

[75] J.S. Cotler et al., Black holes and random matrices, JHEP 05 (2017) 118 [arXiv: 1611.04650] [INSPIRE].

[76] Y. Liu, M.A. Nowak and I. Zahed, Disorder in the Sachdev-Yee-Kitaev model, Phys. Lett. B 773 (2017) 647 [arXiv:1612.05233] [INSPIRE]. 
[77] C. Krishnan, S. Sanyal and P.N. Bala Subramanian, Quantum chaos and holographic tensor models, JHEP 03 (2017) 056 [arXiv: 1612.06330] [INSPIRE].

[78] A.M. García-García and J.J.M. Verbaarschot, Analytical spectral density of the Sachdev-Ye-Kitaev model at finite N, Phys. Rev. D 96 (2017) 066012 [arXiv:1701.06593] [INSPIRE].

[79] T. Li, J. Liu, Y. Xin and Y. Zhou, Supersymmetric SYK model and random matrix theory, JHEP 06 (2017) 111 [arXiv:1702.01738] [INSPIRE].

[80] T. Kanazawa and T. Wettig, Complete random matrix classification of SYK models with $\mathcal{N}=0,1$ and 2 supersymmetry, JHEP 09 (2017) 050 [arXiv:1706.03044] [INSPIRE].

[81] A. Jevicki and B. Sakita, The quantum collective field method and its application to the planar limit, Nucl. Phys. B 165 (1980) 511 [INSPIRE].

[82] A. Jevicki and B. Sakita, Collective field approach to the large- $N$ limit: euclidean field theories, Nucl. Phys. B 185 (1981) 89 [INSPIRE].

[83] S.R. Das and A. Jevicki, Large-N collective fields and holography, Phys. Rev. D 68 (2003) 044011 [hep-th/0304093] [INSPIRE].

[84] I.R. Klebanov and A.M. Polyakov, AdS dual of the critical $O(N)$ vector model, Phys. Lett. B 550 (2002) 213 [hep-th/0210114] [INSPIRE].

[85] R. de Mello Koch, A. Jevicki, K. Jin and J.P. Rodrigues, $A d S_{4} / C F T_{3}$ construction from collective fields, Phys. Rev. D 83 (2011) 025006 [arXiv:1008.0633] [InSPIRE].

[86] R. de Mello Koch, A. Jevicki, J.P. Rodrigues and J. Yoon, Holography as a gauge phenomenon in higher spin duality, JHEP 01 (2015) 055 [arXiv:1408.1255] [INSPIRE].

[87] R. de Mello Koch, A. Jevicki, J.P. Rodrigues and J. Yoon, Canonical formulation of $O(N)$ vector/higher spin correspondence, J. Phys. A 48 (2015) 105403 [arXiv:1408.4800] [INSPIRE].

[88] K. Jensen, Chaos in AdS 2 holography, Phys. Rev. Lett. 117 (2016) 111601 [arXiv: 1605. 06098] [INSPIRE].

[89] J. Maldacena, D. Stanford and Z. Yang, Conformal symmetry and its breaking in two dimensional nearly Anti-de-Sitter space, PTEP 2016 (2016) 12C104 [arXiv:1606.01857] [INSPIRE].

[90] J. Engelsöy, T.G. Mertens and H. Verlinde, An investigation of $A d S_{2}$ backreaction and holography, JHEP 07 (2016) 139 [arXiv:1606.03438] [INSPIRE].

[91] S. Förste and I. Golla, Nearly AdS $S_{2}$ SUGRA and the super-Schwarzian, Phys. Lett. B 771 (2017) 157 [arXiv: 1703.10969] [INSPIRE].

[92] C. Teitelboim, Gravitation and Hamiltonian Structure in Two Space-Time Dimensions, Phys. Lett. B 126 (1983) 41.

[93] R. Jackiw, Lower dimensional gravity, Nucl. Phys. B 252 (1985) 343.

[94] A. Almheiri and J. Polchinski, Models of AdS $S_{2}$ backreaction and holography, JHEP 11 (2015) 014 [arXiv: 1402.6334] [INSPIRE].

[95] G. Mandal, P. Nayak and S.R. Wadia, Coadjoint orbit action of Virasoro group and two-dimensional quantum gravity dual to SYK/tensor models, JHEP 11 (2017) 046 [arXiv: 1702.04266] [INSPIRE]. 
[96] M. Cvetič and I. Papadimitriou, AdS 2 holographic dictionary, JHEP 12 (2016) 008 [Erratum ibid. 01 (2017) 120] [arXiv: 1608.07018] [INSPIRE].

[97] M. Taylor, Generalized conformal structure, dilaton gravity and SYK, JHEP 01 (2018) 010 [arXiv:1706.07812] [INSPIRE].

[98] P. Diaz, S. Das and M. Walton, Bilocal theory and gravity I, arXiv:1609.08631 [InSPIRE].

[99] P. Diaz and S. Das, Bilocal theory and gravity II, arXiv:1705.03893 [INSPIRE].

[100] M. Mezei, S.S. Pufu and Y. Wang, A 2d/1d holographic duality, arXiv:1703.08749 [INSPIRE].

[101] P. Breitenlohner and D. Z. Freedman, Positive energy in Anti-de Sitter backgrounds and gauged extended supergravity, Phys. Lett. B 115 (1982) 197.

[102] A. Jevicki, K. Jin and J. Yoon, $1 / N$ and loop corrections in higher spin $A d S_{4} / C F T_{3}$ duality, Phys. Rev. D 89 (2014) 085039 [arXiv:1401.3318] [InSPIRE].

[103] A. Kitaev, Notes on $\widetilde{\mathrm{SL}}(2, \mathbb{R})$ representations, arXiv:1711.08169 [INSPIRE].

[104] S.R. Das, A. Ghosh, A. Jevicki and K. Suzuki, 1/q expansion of the SYK model, unpublished.

[105] S.R. Das, A. Ghosh, A. Jevicki and K. Suzuki, Space-time in the SYK model, arXiv: 1712.02725 [INSPIRE]. 